\title{
A Theoretical Study of the Effects of Driven Motion On Rotational Correlations of Biological Systems
}

\author{
BETHE A. SCALETTAR, MELVIN P. KLEIN, and JOHN E. \\ HEARST, Department of Biophysics, Department of Chemistry, and \\ Chemical Biodynamics Division Lawrence Berkeley Laboratory, \\ University of California, Berkeley, California 94720
}

\begin{abstract}
Synopsis
We have mathematically analyzed the angular correlation of molecules subject to coupled Brownian torques and driven rotational motion. In our approach a stochastic rotation operator is used to generate the time-dependent orientation of a Brownian particle. All required expectation values can then be computed from just a knowledge of the average of the rotation operator. In particular, the angular correlation function of molecules moving under the influence of both Brownian and driving torques can be shown to exhibit an exponential decay modulated by a periodic term arising from the nonrandom force. The associated power spectrum exhibits a resonance at a frequency characteristic of the driving torque. We discuss DNA/RNA polymerase, muscle, and flagellar systems to which our analysis is applicable.
\end{abstract}

\section{INTRODUCTION}

An accurate picture of macromolecular dynamics is central to a complete understanding of the mechanisms underlying many biological phenomena. All important cellular biochemistry occurs while the interacting biomolecules are subjected to random, solvent-induced Brownian forces. Moreover, coordinated biological processes rely on nonrandom molecular events that couple energy consumption to the generation of directed motion superimposed on the Brownian background.

There are several well-known biological systems that exhibit a combination of random molecular dynamics and repetitive energy dissipative work. The mechanism by which a bacterium propels itself involves the coupling of a motor-directed long-axis rotary motion to Brownian rotational diffusion. ${ }^{1}$ In active muscle the generation of repetitive rotational motion may provide the thrust by which contraction is accomplished. ${ }^{2}$ Several important macromolecular interactions involving nucleic acids have been postulated to involve some sort of repetitive reorientation. The enzyme RNA polymerase could search for its start signal, the promoter, by diffusing down one of the grooves of the DNA molecule. ${ }^{3}$ Moreover, RNA polymerase may actively turn the DNA template during the process of producing an mRNA (transcription) ${ }^{4}$ (see Fig. 1).

The ubiquity of systems that display a coupling of random diffusion to driven rotational motion has inevitably led to an interest in spectroscopic 


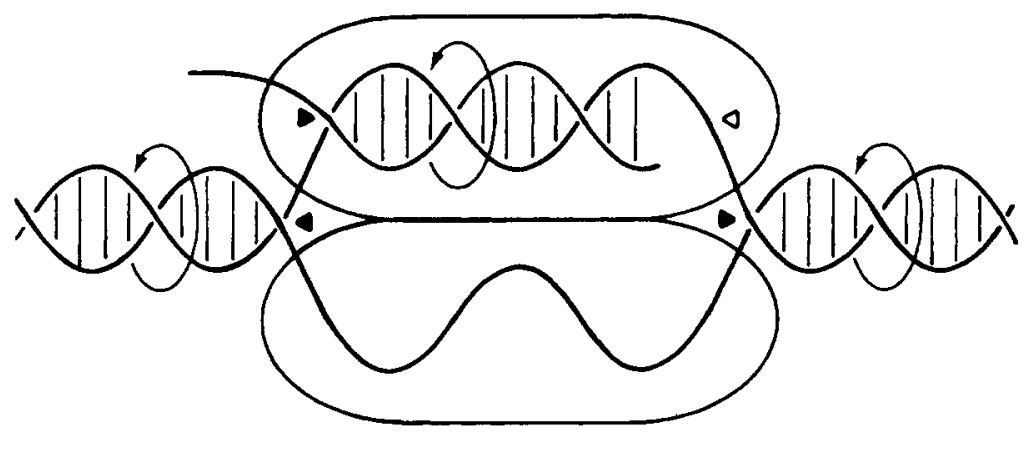

Fig. 1. Propased mechanism underlying the phenomenon of transcription. The enzyme RNA polymerase (depicted by the two ovals) will contact the double-stranded helix (the DNA molecule) and copy the DNA into the single-stranded mRNA. An 18-base region surrounding the point of interaction between the enzyme and the DNA is in a locally unwound state. RNA polymerase inserts a base into the growing mRNA if the hydrogen bonding between the DNA and the mRNA is correct. Therefore, as transcription proceeds the proper orientation of these two molecules must be maintained. Because DNA has a helical structure there must then be a relative rotation of the enzyme/mRNA/DNA complex. It has been suggested that rotation occurs because RNA polymerase exerts a torque on the DNA that causes it to turn. For more details see Ref. 4.

probes for these motions. In order to elucidate the operative biological mechanisms it is desirable to distinguish those components of a complex reorientation that have their origin in random fluctuations from those that are in some sense biologically directed. Correlation measurements are ideally suited to the task of discriminating random macromolecular motions from repetitive reorientation. We have adopted a Langevin-operator approach to address the analysis of rotational correlation in a biological system to purely diffusive and driven rotational motion. We demonstrate that, despite the existence of competing modes of reorientation such as those arising from diffusion, correlation measurements should reveal the presence of driven motion. Specifically, the power spectrum of noise containing a repetitive rotational component should exhibit a resonance at characteristic "forcing" frequencies. For the sake of concreteness we consider a fluorescence correlation experiment, although the concept of modulation is more generally applicable.

\section{THEORY}

In this work we have been primarily motivated by an interest in the motion of DNA in solution. Hence, we consider as our model system a cylindrical DNA subject to an enzymatic torque and solvent-induced Brownian rotation. The DNA is assumed to be labeled with one covalently bound intercalated fluorophore whose transition dipole makes a fixed angle $\beta$ with respect to the long axis of the polymer; the orientation of the DNA is defined by its spherical polar coordinates $\theta_{0}$ and $\phi_{0}$ with respect to a set of lab-fixed axes. It is reasonable to assume that a long macromolecule will have two equivalent diffusion constants that are much smaller than the long-axis rotational diffusion coefficient. Hence, for a system such as an actively transcribing RNA polymerase, rotational diffusion about two axes will be negligible, while there 
will be a coupling of the long-axis rotational diffusion of the enzyme-DNA complex and the driven motion.

In a fluorescence correlation experiment the observed quantity is the autocorrelation function of the fluorescence signal. The autocorrelation function $C(\tau)$ measures the similarity between the fluorescence at a time $t$ and this signal at a later time $t+\tau$. The fluorescence will fluctuate with time because the orientation of the absorption and emission dipoles of the probes will vary with respect to the direction of polarization of the excitation beam and the direction of observation. Changes in dye number will not contribute to the time dependence of the signal because the enzyme-DNA complex translates very slowly relative to its rate of reorientation. It is of course implicit here that the system is small and that therefore the temporal variation in the fluorescence is detectable. This question has been considered in detail elsewhere. ${ }^{5}$

We begin with a brief review of the quantitative relationship between molecular properties and the fluorescence autocorrelation function. A more complete discussion may be found in the article by Aragon and Pecora. ${ }^{6}$ The measured quantity is the fluorescence photocurrent produced when photons impinge on the photocathode of a photomultipler tube and an electron cascade is released. The current from a population of $N$ singly-labeled molecules is given by

$$
i(t)=\operatorname{geQ}(\lambda) \sum_{j=1}^{N} n_{j}(t)
$$

where $g$ is the gain of the photomultiplier, $e$ the electronic charge, $Q(\lambda)$ the efficiency of the photocathode at wavelength $\lambda$, and $n_{j}(t)$ the number of photons emitted per second by the $j$ th fluorophore. The instantaneous deviation of $i(t)$ from the average current $\langle i\rangle$ is $\delta i(t)=i(t)-\langle i\rangle$. The basic quantity of interest is the ensemble averaged (denoted by the angle brackets) autocorrelation function

$$
C(\tau)=\langle\delta i(t) \delta i(t+\tau)\rangle
$$

$C(\tau)$ may be rewritten using the above expression for the photocurrent as

$$
C(\tau)=\langle\delta i(t) \delta i(t+\tau)\rangle=g^{2} e^{2} Q^{2}(\lambda)\left\langle\sum_{j=1}^{N} \delta n_{j}(t) \sum_{i=1}^{N} \delta n_{i}(t+\tau)\right\rangle
$$

This expression simplifies for a dilute solution in which the cross-correlation terms $i \neq j$ may be neglected. If we note that the ensemble average is the same for each molecule

$$
C(\tau)=g^{2} e^{2} Q^{2}(\lambda)\left\langle\sum_{i=1}^{N} \delta n_{i}(t) \delta n_{i}(t+\tau)\right\rangle=N g^{2} e^{2} Q^{2}(\lambda)\langle\delta n(t) \delta n(t+\tau)\rangle
$$

Finally, since the process under consideration is stationary, i.e., invariant 
under time translation,

$$
C(\tau)=N g^{2} e^{2} Q^{2}(\lambda)\langle\delta n(0) \delta n(\tau)\rangle
$$

The number of photons emitted per second by a fluorophore $n(t)$ monitors the orientation of the dye-DNA complex with respect to an external lab frame because it depends on the cosine of the angle between the polarization of the excitation beam and the absorption dipole of the probe. If a particular direction of polarization of the emitted fluorescence is monitored, then the expression for the photocurrent will also contain a term depending on the component of the fluorescence in the direction of detection. In this latter case, sensitivity to orientation is greater, but this extra complexity has no bearing on our basic physical result; hence, we assume isotropic detection of the fluorescence.

The mathematical expression for $n(t)$ is discussed by Aragon and Pecora. They find that the number of photons emitted per second by a fluorophore is given by

$$
n(t)=\frac{q_{f} \epsilon(\lambda) I_{0}}{\tau_{f}} \int_{-\infty}^{t}\left[\vec{m}\left(t^{\prime}\right) \cdot \vec{p}\right]^{2} \exp \left[-\left(\frac{t-t^{\prime}}{\tau_{f}}\right)\right] d t^{\prime}
$$

Here $q_{f}$ is the fluorescence quantum yield of the dye, $\epsilon(\lambda)$ is the extinction coefficient at wavelength $\lambda, I_{0}$ is the incident light intensity, and $\tau_{f}$ is the fluorophore lifetime. The quantity $\vec{m}(t) \cdot \vec{p}$ denotes the dot product of the transition dipole $\vec{m}(t)$ and the constant polarization vector $\vec{p}$. The physical content of Eq. (6) is easy to understand. At time $t$ the number of photons/ second emitted by a dye molecule is given by the integral over all earlier times $t^{\prime}$ of the probability of absorption of the polarized incident light, $\left[\vec{m}\left(t^{\prime}\right) \cdot \vec{p}\right]^{2} I_{0} \epsilon(\lambda)$, multiplied by the probability that a photon absorbed at $t^{\prime}$ will reemitted as fluorescence at time $t, \frac{1}{\tau_{l}} \exp \left[-\left(\frac{t-t^{\prime}}{\tau_{f}}\right)\right]$. In the case of interest here the fluorescence emission is extremely rapid relative to the physical processes under study; hence, it is an excellent approximation to assume that the emission probability is given by its value in the limit that the fluorescence lifetime approaches zero. In this approximation we have

$$
\lim _{\tau_{i} \rightarrow 0} \frac{1}{\tau_{f}} \exp \left[-\left(\frac{t-t^{\prime}}{\tau_{f}}\right)\right]=\delta\left(t-t^{\prime}\right)
$$

and

$$
C(\tau)=N g^{2} e^{2} Q^{2}(\lambda) \epsilon^{2}(\lambda) q_{f}^{2} I_{0}^{2}\left\{\left\langle[\vec{m}(0) \cdot \vec{p}]^{2}[\vec{m}(\tau) \cdot \vec{p}]^{2}\right\rangle-\left\langle[\vec{m}(0) \cdot \vec{p}]^{2}\right\rangle^{2}\right\}
$$

It is clear from expression (8) that orientational information is contained in the autocorrelation function $\left\langle[\vec{m}(0) \cdot \vec{p}]^{2}[\vec{m}(\tau) \cdot \vec{p}]^{2}\right\rangle$. Thus the basic mathematical problem is to follow the evolution of the quantity $\vec{m}(t) \cdot \vec{p}$ in time. The solution is most easily formulated in a coordinate system whose $z$ 


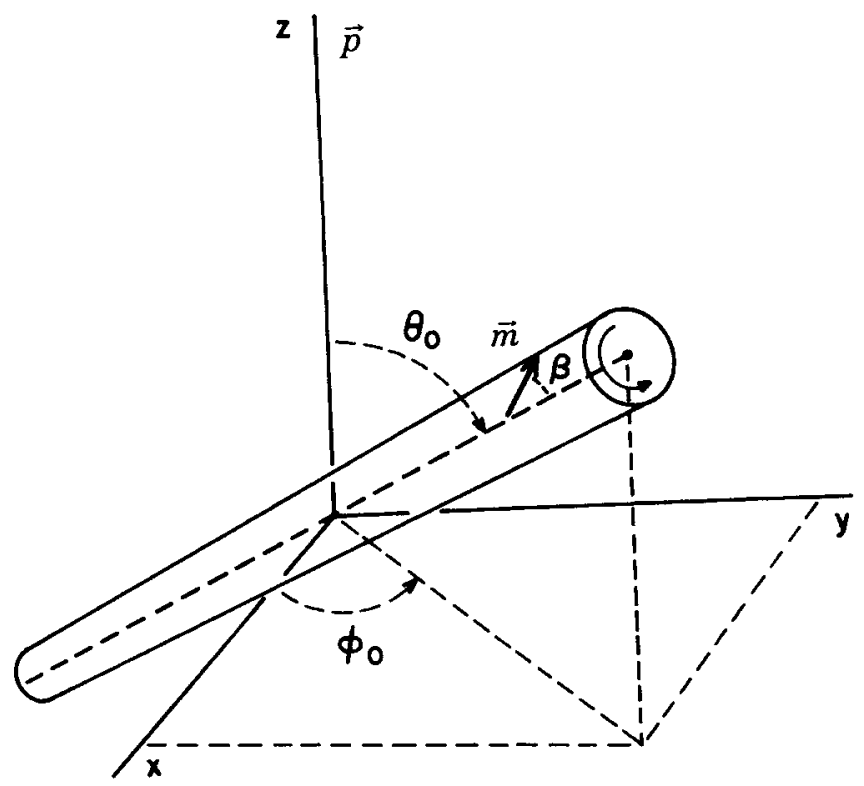

Fig. 2. Coordinate system used to describe the orientation of a rotating cylindrical particle. The absorption dipole $\vec{m}(t)$ makes an angle $\beta$ with respect to the long axis of the polymer. A given DNA molecule has an angular orientation specified by its spherical polar coordinates $\theta_{0}$ and $\phi_{0}$ with respect to the laboratory axes. The polarization of the light, defined by $\vec{p}$, is along the $z$ axis of the laboratory frame. The DNA can undergo coupled rotational diffusion and driven reorientation about its long axis.

axis is along the long axis of the macromolecule. If we denote the angle of rotation of the absorption dipole projection about this $z$ axis in time $t$ by $\theta(t)$ (see Fig. 2) the stochastic behavior of $\vec{m}(t) \cdot \vec{p}$ will be governed by the Langevin equation for $\theta(t)$. The Langevin equation will contain a term representing the Brownian random torque and a driving torque. The external torque is assumed constant; this is probably not an unreasonable model for long-lived rotational phenomena such as flagellar motion or the transcription of DNA by a processive enzyme such as RNA polymerase. We have

$$
I \frac{d w(t)}{d t}=-f w(t)+L(t)+L_{\text {ext }}
$$

Here $f$ is the frictional factor, $I$ is the long axis moment of inertia of a DNA molecule, $L(t)$ is the random torque, $L_{\text {ext }}$ is the constant torque, and $w(t)=d \theta / d t$.

To compute expectation values from Eq. (9) we follow an operator technique for solving the Langevin equation. ${ }^{7}$ The idea is to generate the timedependent orientation of the transition dipole with a rotation operator. Specifically, we write

$$
\vec{m}(t)=R(t) \vec{m}(0)
$$


Here, the rotation operator $R(t)$ is given by the standard matrix expression

$$
R(t)=\left(\begin{array}{ccc}
\cos \theta(t) & -\sin \theta(t) & 0 \\
\sin \theta(t) & \cos \theta(t) & 0 \\
0 & 0 & 1
\end{array}\right)
$$

$R(t)$ is stochastic because its matrix elements are random functions of the time. In fact, by expressing $\vec{m}(t)$ in terms of $\vec{m}(0)$ and the rotation operator, the stochasticity in the problem is entirely confined to $R(t)$. Hence, if we wish to compute ensemble averages of functions of $\theta$, the problem reduces to the determination of the ensemble average of $R(t)$. We now proceed with the computation of $\langle R(t)\rangle$ for a fixed initial orientation of the transition moment. Of course the evaluation of the correlation function requires a second average over the initial distribution; this double average is considered explicitly later.

We move from the Langevin equation of motion for the basic physical variable of interest, in this case the angle $\theta$, to an associated equation for $R(t)$. The equations governing the temporal behavior of these two quantities are related because the matrix $R(t)$ is explicitly defined in terms of $\theta$. If $R(t)$ is given by Eq. (11) then

$$
\frac{d R(t)}{d t}=\left(\begin{array}{ccc}
-\sin \theta(t) & -\cos \theta(t) & 0 \\
\cos \theta(t) & -\sin \theta(t) & 0 \\
0 & 0 & 0
\end{array}\right) \frac{d \theta(t)}{d t}
$$

Setting

$$
J=\left(\begin{array}{ccc}
0 & -i & 0 \\
i & 0 & 0 \\
0 & 0 & 0
\end{array}\right)
$$

We have the evolution equation

$$
\frac{d R(t)}{d t}=-i J w(t) R(t)
$$

Equation (14) for $R(t)$ may be related to the Brownian torque through the equation of motion, that is, the Langevin equation, for $w(t)$. First note that Eq. (9) differs from the undriven Langevin equation by just the constant external torque. It is helpful therefore to reexpress $w(t)$ in terms of quantities characteristic of the simpler problem. If we take the undriven equation, in which $L_{\text {ext }}$ is zero, to have a solution $w_{0}(t)$ then it can be easily verified by substitution into Eq. (9) that

$$
w(t)=w_{0}(t)+\frac{L_{\text {ext }}}{I b}
$$

Here $b=f / I$. 
Upon substituting expression (15) for $w(t)$ into Eq. (14) we have

$$
\frac{d R(t)}{d t}=-i J\left[w_{0}(t)+\frac{L_{\text {ext }}}{I b}\right] R(t)
$$

It is advantageous to make the change of variable

$$
R(t)=S(t) \exp (-i \alpha J t)
$$

where $\alpha=L_{\text {ext }} / I b$. It is apparent that $S(t)$ will satisfy the equation

$$
\frac{d S(t)}{d t}=-i J w_{0}(t) S(t)
$$

Comparison of Eq. (18) with the original evolution Eq. (14) for $R(t)$ shows that $S(t)$ is nothing more than the rotation operator associated with the Langevin equation in the absence of any driving torque. The average of $S(t)$ is known. ${ }^{7}$ The general method used in obtaining the expectation value of $S(t)$ centers around a power series expansion in some small parameter characteristic of the stochastic differential Eq. (18). This approach is outlined in the appendix.

For the stochastic Eq. (18), the power series method of determining the expectation value of an operator yields an exact solution:

$\langle S(t)\rangle=\left(\begin{array}{clr}\exp \{-\gamma[b t-1+\exp (-b t)]\} & 0 & 0 \\ 0 & \exp \{-\gamma[b t-1+\exp (-b t)]\} & 0 \\ 0 & 0 & 1\end{array}\right)$

Here $\gamma=k_{B} T / I b^{2}$. Since $R(t)$ and $S(t)$ differ only by the multiplicative factor

$$
\exp (-i \alpha J t)=\left(\begin{array}{ccc}
\cos \alpha t & -\sin \alpha t & 0 \\
\sin \alpha t & \cos \alpha t & 0 \\
0 & 0 & 1
\end{array}\right)
$$

we obtain the following expression for the expectation value of $R(t)$ :

$$
\langle R(t)\rangle=\exp \{-\gamma[b t-1+\exp (-b t)]\}\left(\begin{array}{ccc}
\cos \alpha t & -\sin \alpha t & 0 \\
\sin \alpha t & \cos \alpha t & 0 \\
0 & 0 & 0
\end{array}\right)+\left(\begin{array}{ccc}
0 & 0 & 0 \\
0 & 0 & 0 \\
0 & 0 & 1
\end{array}\right)
$$

We can now easily determine the temporal behavior of $\langle\sin \theta(t)\rangle$ and $\langle\cos \theta(t)\rangle$. From Eq. (11),

$$
\langle R(t)\rangle=\left(\begin{array}{ccc}
\langle\cos \theta(t)\rangle & -\langle\sin \theta(t)\rangle & 0 \\
\langle\sin \theta(t)\rangle & \langle\cos \theta(t)\rangle & 0 \\
0 & 0 & 1
\end{array}\right)
$$


Upon equating associated matrix elements in Eqs. (21) and (22) we obtain

$$
\begin{aligned}
& \langle\cos \theta(t)\rangle=\exp \{-\gamma[b t-1+\exp (-b t)]\} \cos (\alpha t) \\
& \langle\sin \theta(t)\rangle=\exp \{-\gamma[b t-1+\exp (-b t)]\} \sin (\alpha t)
\end{aligned}
$$

The quantity of real experimental interest is the autocorrelation function of the absorption probability. A determination of this average requires a knowledge of the expectation values of the second powers of cosine and sine of $\theta$. This follows from the matrix expressions for $\vec{m}(t)$ and $R(t)$. We have

$$
\left\langle[\vec{m}(0) \cdot \vec{p}]^{2}[\vec{m}(t) \cdot \vec{p}]^{2}\right\rangle=\left\langle\left[m^{+}(0) H^{+} p\right]^{2}\left[m^{+}(0) R^{+}(t) H^{+} p\right]^{2}\right\rangle
$$

In Eq. (24), $\mathrm{m}^{+}(0)$ and $R^{+}(t)$ denote the transpose of the matrices (written in the molecular frame) representing $\vec{m}(0)$ and $R(t)$ respectively, $p$ is a laboratory referenced column matrix, and $\mathrm{H}^{+}$is the transpose of the matrix that converts the coordinates of a vector in the molecule-fixed axes into the corresponding components in the laboratory frame. We take $\vec{p}$ parallel to the laboratory $z$ axis. An explicit expression for $H$ is

$$
H=\left(\begin{array}{ccc}
\sin \phi_{0} & \cos \phi_{0} \cos \theta_{0} & \cos \phi_{0} \sin \theta_{0} \\
-\cos \phi_{0} & \sin \phi_{0} \cos \theta_{0} & \sin \phi_{0} \sin \theta_{0} \\
0 & -\sin \theta_{0} & \cos \theta_{0}
\end{array}\right)
$$

Once again we note that the angle brackets in Eq. (24) really denote a two time average.

Upon performing the indicated matrix manipulations, the desired autocorrelation function may be expressed as

$$
\begin{aligned}
\left\langle[\vec{m}(0) \cdot \vec{p}]^{2}[\vec{m}(\tau) \cdot \vec{p}]^{2}\right\rangle= & \left\langle\left[-m_{2}(0) \sin \theta_{0}+m_{3}(0) \cos \theta_{0}\right]^{2}\right. \\
& \times\left\{-\sin \theta_{0}\left[\sin \theta m_{1}(0)+\cos \theta m_{2}(0)\right]\right. \\
& \left.\left.+\cos \theta_{0} m_{3}(0)\right\}^{2}\right\rangle
\end{aligned}
$$

Subscripts 1, 2, and 3 in Eq. (26) denote the 1, 2, and 3 components of the vectors relative to the molecular axes. The initial distribution of the projection of $\vec{m}$ onto the plane perpendicular to the long axis of the DNA is assumed uniform. Therefore, when expression (26) is expanded, all terms that involve odd powers of the components of the transition moment average to zero and the correlation function becomes

$$
\begin{aligned}
& \left\langle[\vec{m}(0) \cdot \vec{p}]^{2}[\vec{m}(\tau) \cdot \vec{p}]^{2}\right\rangle \\
& =\left\langle\sin ^{2} \theta\right\rangle\left\langle m_{1}(0)^{2} m_{2}(0)^{2} \sin ^{4} \theta_{0}+m_{1}(0)^{2} m_{3}(0)^{2} \sin ^{2} \theta_{0} \cos ^{2} \theta_{0}\right\rangle_{0} \\
& +\left\langle\cos ^{2} \theta\right\rangle\left\langle m_{2}(0)^{2} m_{3}(0)^{2} \cos ^{2} \theta_{0} \sin ^{2} \theta_{0}+m_{2}(0)^{4} \sin ^{4} \theta_{0}\right\rangle_{0} \\
& \quad+\langle\cos \theta\rangle\left\langle 4 m_{3}(0)^{2} m_{2}(0)^{2} \sin ^{2} \theta_{0} \cos ^{2} \theta_{0}\right\rangle_{0} \\
& \quad+\left\langle m_{3}(0)^{2} m_{2}(0)^{2} \sin ^{2} \theta_{0} \cos ^{2} \theta_{0}+m_{3}(0)^{4} \cos ^{4} \theta_{0}\right\rangle_{0}
\end{aligned}
$$


In Eq. (27) the notation \langle\rangle$_{0}$ explicitly indicates an average over the initial distribution.

It is clear from Eq. (27) that we need to know the expectation values of $\cos ^{2} \theta$ and $\sin ^{2} \theta$. The higher powers of the trigonometric functions may be reexpressed as

$$
\begin{aligned}
& \cos ^{2} \theta=\frac{1}{2}(1+\cos 2 \theta) \\
& \sin ^{2} \theta=\frac{1}{2}(1-\cos 2 \theta)
\end{aligned}
$$

It is now a relatively simple extension of the previous derivation of the averages of $\cos \theta$ and $\sin \theta$ to show that for any $k$

$$
\begin{aligned}
& \langle\cos k \theta\rangle=\exp \left\{-k^{2} \gamma[b t-1+\exp (-b t)]\right\} \cos (k \alpha t) \\
& \langle\sin k \theta\rangle=\exp \left\{-k^{2} \gamma[b t-1+\exp (-b t)]\right\} \sin (k \alpha t)
\end{aligned}
$$

These results can be verified simply by repeating the mathematical steps outlined for the case $k=1$; in this more general situation the relevant matrix generates a rotation by an angle $k \theta$.

To determine the averages indicated in Eq. (27), we require the case $k=2$ :

$$
\begin{aligned}
& \langle\cos 2 \theta\rangle=\exp \{-4 \gamma[b t-1+\exp (-b t)]\} \cos (2 \alpha t) \\
& \langle\sin 2 \theta\rangle=\exp \{-4 \gamma[b t-1+\exp (-b t)]\} \sin (2 \alpha t)
\end{aligned}
$$

Inserting Eq. (30a) into Eq. (27) and explicitly computing the initial averages the correlation function (26) is found to have the form

$$
\begin{aligned}
\left\langle[\vec{m}(0) \cdot \vec{p}]^{2}[\vec{m}(\tau) \cdot \vec{p}]^{2}\right\rangle \propto & \frac{\sin ^{4} \beta}{15} \exp \{-4 \gamma[b \tau-1+\exp (-b \tau)]\} \cos (2 \alpha \tau) \\
& +\frac{4 \sin ^{2} \beta \cos ^{2} \beta}{15} \exp \{-\gamma[b \tau-1+\exp (-b \tau)]\} \\
& \times \cos (\alpha \tau)+\frac{4}{45}\left(\frac{3 \cos ^{2} \beta}{2}-\frac{1}{2}\right)^{2}+\frac{1}{9}
\end{aligned}
$$

It can be shown that $\left\langle[\vec{m}(0) \cdot \vec{p}]^{2}\right\rangle^{2}=\frac{1}{9}$, therefore

$$
\begin{aligned}
C(\tau) \propto & \frac{\sin ^{4} \beta}{15} \exp \{-4 \gamma[b \tau-1+\exp (-b \tau)]\} \cos (2 \alpha \tau) \\
& +\frac{4 \sin ^{2} \beta \cos ^{2} \beta}{15} \exp \{-\gamma[b \tau-1+\exp (-b \tau)]\} \cos (\alpha \tau) \\
& +\frac{4}{45}\left(\frac{3 \cos ^{2} \beta}{2}-\frac{1}{2}\right)^{2}
\end{aligned}
$$


Finally, it is illustrative to examine the Fourier transform of the autocorrelation function (31b). We focus attention on the time-dependent term and compute the power spectrum $J(w)$ as

$$
\begin{array}{r}
J(w)=\int_{0}^{\infty} \cos (w t)\left(\frac{\sin ^{4} \beta}{15} \exp \{-4 \gamma[b t-1+\exp (-b t)]\} \cos (2 \alpha t)\right. \\
\left.+\frac{4 \sin ^{2} \beta \cos ^{2} \beta}{15} \exp \{-\gamma[b t-1+\exp (-b t)]\} \cos (\alpha t)\right) d t
\end{array}
$$

If the exponential in Eq. (32) is expanded in powers of $\exp (-b t)$ and then the integration is performed term by term, it can be demonstrated that

$$
\begin{aligned}
J(w) \propto & \frac{\sin ^{4} \beta \tau_{D}}{30\left[1+\tau_{D}^{2}(w+2 \alpha)^{2}\right]} \\
& +\frac{2 \sin ^{4} \beta \tau_{D} \gamma\left[1+4 \gamma-\tau_{D} \tau_{F}(w+2 \alpha)^{2}\right]}{15\left[1+\tau_{D}^{2}(w+2 \alpha)^{2}\right]\left[(1+4 \gamma)^{2}+\tau_{F}^{2}(w+2 \alpha)^{2}\right]}+\cdots \\
& +\frac{2 \sin ^{2} \beta \cos ^{2} \beta \tau_{D}}{15\left[1+\tau_{D}^{2}(w+\alpha)^{2}\right]} \\
& +\frac{2 \sin ^{2} \beta \cos ^{2} \beta \tau_{D} \gamma\left[1+\gamma-\tau_{D} \tau_{F}(w+\alpha)^{2}\right]}{15\left[1+\tau_{D}^{2}(w+\alpha)^{2}\right]\left\{[1+\gamma]^{2}+\tau_{F}^{2}(w+\alpha)^{2}\right\}}+\cdots
\end{aligned}
$$

In Eq. (32) $\tau_{D}=f / k_{B} T$ and $\tau_{F}=(I / f)$. $J(w)$ also contains four other terms that can be obtained by replacing the quantity $\alpha$ by $-\alpha$ in expression (33).

\section{DISCUSSION}

The fluorescence photocurrent autocorrelation function arising from a dilute sample of cylindrical molecules subject to random Brownian and constant external torques

$$
\begin{aligned}
C(\tau) \propto & \frac{-\sin ^{4} \beta}{15} \exp \{-4 \gamma[b \tau-1+\exp (-b \tau)]\} \cos (2 \alpha \tau) \\
+ & \frac{4 \sin ^{2} \beta \cos ^{2} \beta}{15} \exp \{-\gamma[b \tau-1+\exp (-b \tau)]\} \cos (\alpha \tau) \\
& +\frac{4}{45}\left(\frac{3 \cos ^{2} \beta}{2}-\frac{1}{2}\right)^{2}
\end{aligned}
$$

exhibits an exponential decay that is modulated by a cosine term. The 
modulation frequency is $\alpha=L_{\text {ext }} / I b$. The exponential contribution to the correlation function arises from the random Brownian torque; in fact, by setting $L_{\text {ext }}=0$, we obtain the purely exponential decay corresponding to the undriven case

$$
\begin{aligned}
C(\tau) \propto & \frac{\sin ^{4} \beta}{15} \exp \{-4 \gamma[b \tau-1+\exp (-b \tau)]\} \\
& +\frac{4 \sin ^{2} \beta \cos ^{2} \beta}{15} \exp \{-\gamma[b \tau-1+\exp (-b \tau)]\}+\frac{4}{45}\left(\frac{3 \cos ^{2} \beta}{2}-\frac{1}{2}\right)^{2}
\end{aligned}
$$

The oscillatory terms, on the other hand, are manifestations of the correlation that would be observed from molecules moving under the influence of constant and frictional torques. Consider the equation of motion of a cylinder in the absence of random torques:

$$
I \frac{d w}{d t}=-f w(t)+L_{\mathrm{ext}}
$$

Under steady state conditions the angular acceleration is equal to zero, and the steady state angular velocity has the form

$$
\begin{gathered}
f w_{s s}=L_{\mathrm{ext}} \\
w_{s s}=\frac{L_{\mathrm{ext}}}{f}
\end{gathered}
$$

The modulation frequency appearing in Eq. (34), $\alpha=L_{\text {ext }} / I b=L_{\text {ext }} / f$, is the steady state angular velocity. Hence, the sinusoidal term in Eq. (34) gives the correlation that would be observed from a molecule subject to frictional and constant torques, under steady state conditions. The second harmonic arises because the expression for the probability of absorbing polarized light depends on the quantity $[\vec{m}(\tau) \cdot \vec{p}]^{2}$; there is, therefore, a $2 w_{s s}$ periodicity associated with antiparallel orientations of the transition dipole.

If the Fourier transform of the correlation function, the power spectrum, (33) is examined, it is apparent that there are peaks or resonances in the spectrum at $\pm w_{s s}$ and $\pm 2 w_{s s}$. Thus, characteristic driving frequencies are also manifest in the power spectrum of the signal.

Finally, we note that long cylindrical molecules such as DNA exhibit internal bending and torsional motions that will affect the fluorescence photocurrent autocorrelation function. ${ }^{8}$ As long as these motions are of restricted amplitude they should not destroy the long-time reorientational correlations discussed here. We are now in the process of analyzing the effects of constrained internal motions on the FCS signal. 


\section{Relationship to Other Work}

Angular correlation in a system subject to coupled Brownian rotational diffusion and driven reorientational motion has been addressed from a different mathematical viewpoint in a recent publication. ${ }^{9}$ The authors adopt the more traditional generalized Fokker-Planck (FP) approach to the problem of Brownian motion and derive from the FP equation the required expectation values. The diffusion equation method has also been used to demonstrate the Doppler shift resonance phenomenon in systems exhibiting coupled Brownian translational motion and directed flow. ${ }^{10}$

The rotational results obtained from the FP partial differential equation yield a power series solution for the autocorrelation function if the labeled molecule is allowed to diffuse in three dimensions. ${ }^{9}$ The solution obtained from the Langevin-operator approach used here is exact and equivalent to that obtained from an FP approach when only a single diffusion constant is relevant. The consistency of the FP and Langevin results is a nice example of the equivalence of the partial and stochastic differential equation approaches to the problem of Brownian motion.

In this work we emphasize an essential physical point: In a rotationally driven system there is a periodic modulation of the pure Brownian correlation; this modulation is manifest as a resonance in the power spectrum of the signal. From the Langevin viewpoint we also obtain additional physical insight by identifying the resonant frequency as the steady state angular velocity of a molecule subject to purely frictional and driving torques.

This work was supported by National Institutes of Health Grant GM30781 and by the Office of Energy Research, Office of Health and Environmental Research of the U.S. Department of Energy under contract number DE-AC03-76SF00098. We wish to thank Robert Harris and the referees for helpful suggestions. We also thank James Abney and Richard Scalettar for their continual support. This paper is dedicated to the memory of Florence C. Scalettar.

\section{APPENDIX}

Suppose a general stochastic quantity of interest, $X(t)$, is governed by some evolution equation:

$$
\frac{d X(t)}{d t}=\epsilon O(t) X(t)
$$

Here, $\epsilon$ is a small parameter and $O(t)$ is some operator. It is possible to expand $X(t)$ as

$$
X(t)=\sum_{i} \epsilon^{i} f^{i}(t)\langle X(t)\rangle
$$

Here, the $f^{i}(t)$ are stochastic operators that satisfy $\left\langle f^{i}(t)\right\rangle=0$. The mean value of $X(t)$ will satisfy some nonstochastic differential equation of the form

$$
\frac{d\langle X(t)\rangle}{d t}=\sum_{i} \epsilon^{i} \Omega^{i}(t)\langle X(t)\rangle
$$

Here the $\Omega^{i}(t)$ are nonstochastic: they are equal to their average.

One may find expressions for the $\Omega^{i}(t)$ by substituting the power series for $X(t)$ and $\langle X(t)\rangle$ into the basic evolution equation for $X(t)$ and then equating powers of $\epsilon$. The general expressions 
for the expansion coefficients in terms of integrals over operator $O(t)$ are given in Ref. 7 . It is shown that

$$
\begin{gathered}
\Omega^{1}(t)=\langle O(t)\rangle \\
\Omega^{2}(t)=\int_{0}^{t}\left(\left\langle O(t) O\left(t_{1}\right)\right\rangle-\langle O(t)\rangle\left\langle O\left(t_{1}\right)\right\rangle\right) d t_{1}
\end{gathered}
$$

The expressions for the higher expansion coefficients $\Omega^{i}(t)$ are more complicated functions of the operator $O(t)$, but in general the $\Omega^{i}(t)$ are expressible in terms of integrals over ensemble-averaged products of $O(t)$. The $\Omega^{i}(t)$ are evaluated for a specified $O(t)$. Once the $\Omega^{i}(t)$ are determined the equation of motion for $\langle X(t)\rangle$ may be integrated to find the ensemble average of $X(t)$.

\section{References}

1. Berg, H. C., Manson, M. D. \& Conley, M. P. (1982) in Prokaryotic and Eukaryotic Flagella, Amos, W. B. \& Duckett, J. D., Cambridge University Press, Cambridge, p. 1.

2. Borejdo, J., Putnam, S. \& Morales, M. F. (1979) Proc. Natl. Acad. Sci. USA 76, 6346-6350.

3. Schurr, J. M. (1979) Biophys. Chem. 9, 413-414.

4. Gamper, H. \& Hearst, J. E. (1982) Cell 29, 81-90.

5. Elson, E. L. \& Magde, D. (1974) Biopolymers 13, 1-27.

6. Aragon, S. R. \& Pecora, R. (1975) Biopolymers 14, 119-138.

7. McConnell, J. (1980) Rotational Brownian Motion and Dielectric Theory, Academic Press, New York, p. 136.

8. Shibata, J. H., Fujimoto, B. S. \& Schurr, J. M. (1985) Biopolymers 24, 1909-1930.

9. Hoshikawa, H. \& Asai, H. (1985) Biophys. Chem. 22, 167-172.

10. Berne, B. J. \& Pecora, R. (1976) Dynamic Light Scattering, Wiley Interscience, New York, p. 74 .

Received October 6, 1986

Accepted February 25, 1987 